Rapport - Société canadienne d'histoire de l'Église catholique

\title{
La vision religieuse de Champlain
}

\section{Raymond Barbeau}

Volume 28, 1961

URI : https://id.erudit.org/iderudit/1007378ar

DOI : https://doi.org/10.7202/1007378ar

Aller au sommaire du numéro

Éditeur(s)

La Société canadienne d'histoire de l'Église catholique

ISSN

0318-6148 (imprimé)

1927-7075 (numérique)

Découvrir la revue

Citer cet article

Barbeau, R. (1961). La vision religieuse de Champlain. Rapport - Société

canadienne d'histoire de l'Église catholique, 28, 53-62.

https://doi.org/10.7202/1007378ar

Tous droits réservés @ La Société canadienne d'histoire de l'Église catholique, 1963
Ce document est protégé par la loi sur le droit d'auteur. L’utilisation des services d'Érudit (y compris la reproduction) est assujettie à sa politique d'utilisation que vous pouvez consulter en ligne.

https://apropos.erudit.org/fr/usagers/politique-dutilisation/ 


\section{La vision religieuse de Champlain}

La nation canadienne-française réfléchit beaucoup de ce temps-ci sur ses destinées et je suis persuadé qu'elle s'orientera sur la bonne voie. Au cours de mes recherches d'honnête homme sur l'histoire du Québec, j'ai souvent été étonné de constater l'intervention divine au moment où tout paraissait perdu. J'ai voulu étudier de plus près la vie et l'œuvre du Père de la Nouvelle-France pour mieux comprendre ses intentions. Ce sera la réponse de l'histoire à ceux qui désespèrent de notre peuple aussi bien qu'à ceux qui tentent de réduire les dimensions de notre foi ou de neutraliser nos institutions, contrairement aux intentions des fondateurs de notre pays. Puisse Champlain nous donner une leçon de courage !

Ma communication, forcément succincte, vous rappellera l'histoire passionnante de Samuel de Champlain, sous l'angle religieux. Premièrement, ce qu'a été la vision religieuse de Champlain; deuxièmement, de quels obstacles elle a été traversée; troisièmement, dans quelles œuvres elle s'est réalisée : voilà ce que j'essayerai de vous dire au meilleur de mon information.

\section{I - Ce qu'a été la vision religieuse de Champlain}

Remarquons d'abord que la pensée de Champlain est un tout. Il est aussi grand chretien que grand politique. Sa ferveur religieuse couronne son amour de la patrie. Il accomplira une grande ouvre. Il ne le sait pas encore parfaitement lorsqu'il fonde Port-Royal le 16 juin 1604, car il est alors plus mordu de l'esprit de la découverte que du génie de la colonisation, et il voudrait bien trouver le premier le passage vers la Chine, ou tout au moins prolonger de vastes explorations côtières vers la Floride. Il connaîtra parfaitement sa vocation le 30 juin 1608, quand il fonde Québec sur les bords du Saint-Laurent. A ce moment-là, il est en possession d'une vision coloniale complète, et c'est celle d'une Nouvelle-France à établir effectivement sur cet immense territoire. Il ne s'agit plus de découvrir seulement; il ne s'agit plus d'exploiter commercialement la traite des fourrures; il s'agit de construire un établissement durable, de créer avec des éléments humains, matériels et moraux une patrie nouvelle à l'image de la mère patrie. Il s'agit d'engendrer une société nouvelle, capable de soutenir une humanité nouvelle. Il met ses ouvriers à l'œuvre pour construire la fameuse "Abitation de Quebecq".

Champlain veut donner en Amérique un empire à son Roi. Cela semble, à première vue, une visée purement politique. Mais nous sommes à une époque où l'idée religieuse est partie intégrante de l'idée politique. On n'a pas encore dépecé l'homme en homo economicus, 
homo politicus et homo religiosus. Il règne une unité de l'homme qui ne s'accommode pas du couteau scientifique et positiviste. L'homme est encore un animal raisonnable habité de l'Esprit de Dieu.

Pour sa part, le Roi de France est appelé le Roi Très-Chrétien, et s'il commet parfois des péchés mortels à l'encontre de son nom, il sait les accuser devant son Dieu et proposer quand même à ses peuples, outre le salut du corps, le salut de l'âme. Il se range donc à l'obéissance de l'Eglise, et cette humilité lui obtient l'obéissance de ses sujets. Le cas typique est celui d'Henri IV le Rni qui fonda Québec... comme point culminant de son règne! La Nation lui avait imposé la foi catholique. Cette conviction plutôt extérieure ne l'empêcha pas de marcher, à la tête du peuple français, comme un fils aîné de l'Eglise. Fait à remarquer : parmi les Ligueurs qui lui avaient imposé la foi catholique, apostolique et romaine, se trouvait un gentilhomme saintongeois, sergent de cavalerie aux armées de la Ligue et portant le nom de Samuel de Champlain! Henri IV engendra Louis XIII, réplique de saint Louis à l'aurore du XVII' siècle. Et Louis XIII prit comme ministre un évêque de l'Eglise de Rome : Armand Duplessis, évêque de Luçon, cardinal duc de Richelieu. Si Richelieu fut moins « catholique " que son roi, si même il contribua à la dissociation du principe religieux et du principe temporel dans la grande politique, il a toujours compris que les affaires du royaume allaient d'autant mieux que les affaires de Dieu dans le royaume prospéraient davantage.

Toujours pour cerner la vision religieuse de Champlain, rappelons le mouvement de rénovation qui marqua le catholicisme français au début du $\mathrm{XVII}^{\circ}$ siècle. Quelques noms en diront plus que de longues dissertations : à la date où nous sommes, 1608, François de Sales a 41 ans; Vincent de Paul, 32; Pierre de Bérulle, fondateur de l'Oratoire, 33. En plus, la Compagnie de Jésus, chassée de France par Henri IV en 1595 (le roi avait fait pendre le Père Guignard pour un pamphlet du temps de la Ligue), avait été rappelée par lui en 1603; et cette même année, Henri donnait à la Compagnie le magnifique collège qu'il venait de fonder à La Flèche et gardait pour éclairer sa conscience souvent orageuse le désormais célèbre Père Coton. Au passage, disons qu'Henri avait une véritable tendresse pour François de Sales et qu'il fit cinq fois des instances pour le retenir à la Cour; rappelons aussi que c'est vers les mêmes dates que Vincent de Paul devint aumônier de la reine, femme d'Henri IV.

Jusque-là, dit un historien de l'Eglise, il manquait à la religion française l'élan mystique. Sous l'influence des nouveaux docteurs spirituels, on entra dans l'amour de Dieu, et de là on passa au dévouement sans bornes pour le prochain.

Ce fut l'origine, en 1627, de la Compagnie du Saint-Sacrement, association laïque pour faire aimer Dieu et le prochain. Or il se trouve que le fondateur de cette association, Henri de Lévis, duc de Ventadour, fut donné à Champlain par Richelieu comme vice-roi de la Nouvelle-France, où il envoya à ses frais les six premiers jésuites.

Il est temps que nous arrivions directement à la pensée de Champlain lui-même. Cette pensée, nous la déduirions de sa conduite, mais 
elle nous est connue aussi, fort heureusement, par ses écrits qui forment deux volumes. Les récits de voyage de Champlain entrent dans cette littérature coloniale qui exerça une influence prépondérante sur la création des colonies françaises. La poésie elle-même s'en mêlait. A Port-Royal, en 1606 et 1607, les Muses faisaient des odes et des sonnets, par la bouche de l'avocat parisien Marc Lescarbot, venu là a pour fuir un monde corrompu ". Le premier ouvrage publié par Champlain - c'est en 1613, pas à Québec mais à Paris - s'ouvre de même par une longue pièce de vers, où nous allons apprendre en discours indirect les intentions du Père de la Nouvelle-France. Ecoutez ces alexandrins assez proches de la prose mais découvrant le cœur :

Il nous promet encor de passer plus avant,

Réduire les gentils et trouver le Levant,

Par le Nord ou le Sud, pour aller à la Chine.

C'est charitablement tout pour l'amour de Dieu.

"Charitablement, tout pour l'amour de Dieu ": un disciple de François de Sales, ou de Bérulle, ou de Vincent de Paul, un sociétaire de la Compagnie du Saint-Sacrement ou un prosélyte des grands docteurs jésuites de l'époque n'aurait pas parlé autrement. Le Père Coton, propre confesseur du roi, ne proposait-il pas cette totalité, cette dimension surhumaine de l'amour quand il disait : "Allons à Dieu, mon âme, en démarche de géants!

Nous avons lu : "réduire les gentils". Cela peut vouloir dire les dompter et même les anéantir. Cortez l'avait fait au Mexique et Pizarre au Pérou. Dans son livre publié en 1619, Champlain explique en prose ce mot fort du poème. Il dit à Louis XIII, dans l'épître dédicatoire : «C'est une grâce spéciale de Dieu, d'avoir voulu réserver sous votre règne l'ouverture de la prédication de l'Evangile et la connaissance de son saint nom à tant de nations qui n'en avaient jamais ouï parler. " Réduire les gentils, c'est donc conduire les infidèles, les sauvages, à la connaissance du vrai Dieu.

Si nous voulons connaître la pensée de Champlain par la comparaison des fins coloniales, c'est-à-dire dans le rapport des fins politiques et des fins religieuses, nous avons de lui cette parole souvent citée, démonétisée par l'usage, mais issue sans doute du fond de son âme et qui fera à jamais son honneur à lui et l'honneur de l'âme française : - Le salut de l'âme d'un Indien vaut mieux que la conquête d'un empire. 》

C'est le lieu de nous demander d'où avait pu venir à notre héros cette élévation de pensée.

La première réponse est toujours dans le caractère et la libre volonté de l'homme. Cortez voulait de l'or; Richelieu pouvait vouloir de la puissance; Champlain voulait des âmes : il voulait d'abord le royaume de Dieu. Ce secret du cœur est avant tout une détermination personnelle et le mystère du don de Dieu pour chaque homme.

Néanmoins des circonstances interviennent. Nous avons vu celles du milieu spirituel français de l'époque. Il faut certainement faire état 
du milieu où Champlain vécut son destin. C'est cette population sauvage qui l'entoura pendant trente ans. Dès la première heure, elle lui apparut selon ce qu'elle était : misérable de sa double ignorance, humaine et divine. L'expression revient sans cesse sous sa plume pour ce qui est de l'ignorance spirituelle : "Ces peuples malheureux qui n'auront jamais connu le vrai Dieu. "Champlain s'éprit à la fois d'amitié humaine et d'amitié divine pour ces sauvages. Rien de plus touchant d'abord que l'estime, la considération, le respect qu'il leur accordait. Des chefs montagnais, algonquins et hurons, il se fait de vrais amis, de véritables dévots à sa personne. Il les raisonnait, discutait doucement avec eux, s'émerveillait même, malgré leur ignorance, de la force et de la souplesse de leur esprit. Religieux comme il l'était lui-même, comment n'aurait-il pas estimé que le plus grand bien à leur transmettre était la foi ? Champlain n'est pas un théoricien de la colonisation, assis à douze cents lieues dans un bureau métropolitain. Il a autour de lui la matière vivante sur laquelle il s'agit de travailler, qu'il s'agit de sculpter en formes dignes de l'humanité. Et cette matière n'est pas avant tout le colon mais l'indigène. D'ailleurs, il y a si peu de colons dans l'ambiance du gouverneur de la NouvelleFrance : 200 à sa mort, c'est-à-dire trente-deux ans après le début de l'entreprise française! C'est en ce sens qu'on peut dire que Champlain est un chef de missionnaires plus qu'un organisateur de colonie. Je pense que si on l'avait écouté, il aurait créé avec les indigènes d'Amérique l'empire qu'il désirait pour la France, qu'à sa mort, le 25 décembre 1635, au lieu de ces deux cents colons françaic, nous aurions eu au Saint-Laurent peut-être cinquante mille braves habitants hurons, iroquois, montagnais, iroquois même, rangés sous nos lois et sous les lois du Dieu d'amour. A travers cela, un ou deux milliers de Français pour civiliser et sanctifier ces bien-aimés barbares!

\section{$\mathrm{Au}$ fait, on a un peu écouté Champlain.}

La solidarité de l'idée colonisatrice et de l'idée missionnaire, restée le fondement du système français pendant au moins tout le $\mathrm{XVII}^{\circ}$ siècle, appartient avant tout à Champlain. Sans doute les missionnaires, Récollets d'abord puis Jésuites, ont soutenu ce principe, en ont propagé le rayonnement. Mais c'est bien à l'obstiné créateur de la colonie qu'on doit en reconnaître avant tout la paternité et c'est à lui qu'on doit en attribuer l'acceptation par la métropole.

L'historien de la France coloniale d'Ancien Régime, F. Saintoyant, voulant résumer l'œuvre de Richelieu dans le domaine des colonies, dit expressément : " Il en vit les aspects politique, économique et sociaux; il partagea les idées de Champlain sur la nécessité de s'assurer la collaboration des indigènes, etc. "Quand Richelieu décida de prendre lui-même en mains la grande œuvre et qu'il mit sur pied la Compagnie des Cent-Associés, il ne fit pratiquement que traduire, dans l'organisation de la Compagnie aussi bien que dans les documents d'Etat qui l'instituaient, les idées maîtresses de Champlain. Richelieu ne faisait rien de grave en politique sans procéder d'abord à de grandes enquêtes..., comme nous. Pour la Nouvelle-France, les témoins étaient Champlain, les missionnaires, les marchands. La théorie mercantile 
donnait le pas à la traite des pelleteries et aux pêcheries; elle excluait le peuplement comme un mal et la mise en valeur de la colonie comme une concurrence. Richelieu n'exclut pas le commerce, mais il le mit au troisième rang, après l'extension de la foi et l'établissement des colons à demeure. Tel est le programme de la charte des CentAssociés (1627).

Si on se reporte au règne suivant, l'idéal est maintenu. Louis XIV et Colbert le reprennent sans changement. La charte de la Compagnie des Indes Occidentales, en 1664, dira, par exemple : « Nous regardons dans l'établissement des dites colonies principalement la gloire de Dieu en procurant le salut des Indiens et sauvages. \$

Il faut bien dire quelques mots ici de l'exclusive prononcée par la métropole contre les protestants en Nouvelle-France. Une encre généreuse s'est dépensée pour voir là une erreur capitale de la France colonisante. Si c'est une erreur, il faut en attribuer le mérite à Champlain. D'abord pour des raisons fort pratiques. Quand on fonda l'Acadie, l'interdit n'avait pas encore été prononcé. C'était sous Henri IV, qui ne malmena jamais trop ses anciens coreligionnaires. Et en Acadie, la colonie se trouvait pourvue d'un pasteur calviniste et d'un prêtre catholique. Champlain les surprit un jour réglant leurs débats théologiques à coups de poings ! Depuis ce temps-là, il entretint des doutes sur le bi-religionnisme. Pour fonder Québec, il dut monter des bateaux de commerce que gouvernaient des entrepreneurs huguenots. Nouveaux embarras. Discordes parmi le personnel bi-religionnaire des navires et des magasins. Contradictions devant les sauvages entre les prédications des deux groupes. Champlain se convainquit qu'il ne pouvait y avoir de plus grand obstacle à l'évangélisation que cette lutte d'influence.

Il sera intéressant, avant de quitter ce point, de comparer un peu la vision religieuse du fondateur de Québec à celle du fondateur de Montréal. Comment furent-elles différentes? Comment se ressemblèrent-elles?

Apparemment, Maisonneuve ne fondait qu'un poste tandis que Champlain fondait un royaume. A la vérité, les deux fondaient le royaume. Vous savez bien que de tout temps Montréal déteignit sur Québec, fortifia Québec, souvent sauva Québec et toute la NouvelleFrance, comme en 1660 ! Maisonneuve prit la Nouvelle-France, sept ans après, où Champlain l'avait laissée. De ce chef, les deux jumeaux, aussi beaux et magnanimes l'un que l'autre, avaient bu le même lait de la Mère Eglise, de la Mère Eglise de France, et voulaient fermement le règne du Christ qui aime les Francs. Ils le voulaient avec ferveur; ils le voulaient en apôtres, en missionnaires.

La différence semble reposer sur le fait que l'intention de Champlain était plus politique que celle de Maisonneuve, comme d'ailleurs l'était sa commission de lieutenant général pour le Roy en NouvelleFrance. Maisonneuve fondait une cité mystique et missionnaire; Champlain, une cité politique et missionnaire. Chez Champlain, vous trouvez une haute ascèse : les missionnaires nous ont dit comment étaient 
réglés les exercices de piété à l' * Abitation ». Chez Maisonneuve, vous trouvez tous les traits du chevalier mystique, du chrétien qui ambitionne pour lui-même la haute contemplation et qui cherche à en partager le bienfait avec les siens. Champlain est un actif qui contemple; Maisonneuve est un contemplatif qui agit. Tous tant que nous sommes, nous avons sans doute hérité, selon nos régions, de l'une ou de l'autre tradition! Mais elles ont entre elles beaucoup d'harmonies, beaucoup, comme disent les musiciens, de notes communes...

\section{İ̄ - Les obstacles trouves sur le chemin}

Plus une idée est belle, plus elle semble difficile à véhiculer. Nous pouvons croire, à trois siècles et demi de distance, sur la foi de pâles résumés, que l'idée vraiment grandiose de Champlain fut portée par des anges sur le promontoire de Québec et de là à mille milles jusqu'au fond de la Huronie et même, par des anges coureurs de bois jusqu'au fond du lac Supérieur. Pour nous détromper, il faut lire une monographie détaillée. Je vous conseille, excusez ma présomption, celle qu'a écrite Emile Micard pour la collection Les grands navigateurs, et qui s'intitule l'Effort persévérant de Champlain (Ed. Pierre Roger, Paris, 1929). L'impression qui nous en reste est celle d'une écrasante mélancolie. Vous voyez cet homme piétiner sur place, reculer de plusieurs pas quand il en a fait péniblement un ou deux, persévérer, oui, dans son effort, mais finalement toucher le terme de son existence sans avoir, semble-t-il, rien fait qui vaille. J'ai donné tout à l'heure la statistique en hommes de ce qu'il a réussi : deux cents âmes pour toute la Nouvelle-France; résultat d'un effort de trente années !

\section{Qu'est-ce donc qui entravait la réalisation de ses projets?}

Commençons par le principal et le plus banal obstacle : le démon. Si cette bête rôde sans cesse autour de nous cherchant à nous dévorer, elle rôdait très fort en Amérique quand les envoyés de Dieu apparurent. La barrière que les Iroquois opposèrent à toute avance de l'évangélisation, non seulement chez eux, mais dans tout l'ensemble du territoire, leurs opérations de destruction massive contre les nations devenues accueillantes à la prédication chrétienne, leur haine sans merci contre les Français, tout cela ne s'explique bien que par des influences sataniques. Combien de fois les missionnaires n'ont-ils pas attesté qu'ils sentaient cette présence de Belzébuth dans le grand corps des tribus comme on la sent dans le corps d'un possédé! Ils n'ont jamais douté de la communication des sorciers, même hurons, avec l'Esprit du mal.

Après les démons, mentionnons logiquement les bons diables. Ce sont tous ceux qui ont une petite foi dans les grandes choses, petite foi nationale ou religieuse. Voyez Sully, par exemple, l'illustre ministre d'Henri IV. Qu'est-ce que peut faire un roi quand son ministre a l'âme froide? Henri IV s'enflammait pour Champlain, à qui il souhaita deux ou trois fois bon voyage à la porte de sa chambre. Mais Sully, on le sait, ne croyait pas à l'œuvre coloniale et ne croyait pas même à l'aptitude du Français pour l'œuvre coloniale ! Et Port-Royal, puis Québec, restaient de petits points noirs sur une carte... 
Appellerons-nous aussi bons diables les marchands? Champlain n'est pas éloigné de les appeler des démons. S'il traverse la mer vingtquatre fois pour le service de Port-Royal ou de Québec, c'est surtout pour dire à la Cour : "Délivrez-nous des marchands ", comme on dit dans le Pater : " Délivrez-nous du mal ! \& Les marchands, à certaines années, servent des dividendes de $40 \%$ à leurs actionnaires grâce au pactole du castor. Mais pour cela, il ne faut pas que les sauvages soient établis : il faut qu'ils restent à chasser dans les bois; pour cela, il ne faut pas que les Français abattent la forêt, grande chasse gardée, et que l'habitant partage avec le marchand le bénéfice de la traite; et pour le succès du commerce, qu'avons-nous à faire de l'Evangile ? Champlain n'exclut pas le commerce au même degré que la Compagnie de Notre-Dame de Montréal et Maisonneuve; il croit par moment pouvoir apprivoiser cette passion plus vivace que la passion apostolique. Il fait raturer une compagnie de commerce pour en établir une qui serait meilleure, c'est-à-dire un peu plus désintéressée, mais à chaque fois, il y perd sa peine et une illusion de plus. Quand en 1627, Richelieu en personne établit la Compagnie des Cent-Associés et s'inscrit en personne en tête des actionnaires, tout semble sauvé. La Compagnie semble créée pour le Sauvage, au point que l'un de ses statuts donne d'emblée la pleine citoyenneté française à tout indigène baptisé. La Compagnie doit pourvoir aussi à l'établissement massif de colons. Le commerce ne sera que l'outil : il arrive en dernier lieu dans la mention des privilèges de la charte. C'est ce qu'on trouve dans les documents. Dans la réalité, les Cent-Associés n'en vaudront pas dix, et ces dix, qui ne bougent d'ailleurs pas de la métropole, continueront de penser à leur portefeuille plus qu'au salut des âmes ou même à l'établissement d'un empire.

Il est vrai qu'une autre espèce de bons diables se seront mis en travers, et ce seront les Anglais.

L'instant le plus mélancolique de la vie de Champlain est celui où il dut remettre, en 1629, la clé du fort Saint-Louis à David et Thomas Kirke. Il laissait là Couillard et Hébert, et il partait lui-même avec sa mince équipe de missionnaires, après avoir fermé la porte à l'Evangile. Le premier convoi de la Compagnie des Cent-Associés avait été pris par l'ennemi et la Nouvelle-France avait dû être livrée. Champlain passa trois ans à redemander son domaine. Il le recouvra anéanti et passa les trois dernières années de sa vie à rebâtir à partir du néant !

C'est de martyre à petit feu qu'il faut parler à propos de cette carrière d'apôtre, toute fondue à celle d'un grand colonisateur. Emile Micard admire la grandeur de la vie de Champlain : « Peu de vies ont été aussi grave que celle de ce soldat qui consacra trente-cinq ans toute sa vie d'homme - à sa tâche d'explorateur et de colon, trentecinq ans coupés par vingt-quatre voyages en France, mais quels voyages! pour quémander les secours, démasquer des traîtres, dénouer des intrigues et généralement sans succès. " Champlain aurait eu le droit de finir avec une population néo-française de 15,000 âmes pour le moins, avec une conquête évangélique et civilisatrice d'un nombre 
d'âmes au moins égal. Il accepta sa petite réussite. " Il eut une âme simple et pieuse, dit encore le même biographe, une âme nourrie des idées régnantes, résignée à l'inévitable, qu'elle considérait comme l'effet de la volonté divine.

\section{III - L'œuvre apostolique de Champlain}

J'aurai donc bien peu de choses à dire sur l'œuvre dans laquelle Champlain a concrétisé sa vision religieuse. Cette œuvre se résume à la dispersion de quelques minuscules grains de semence.

Champlain lui-même a été missionnaire. Vous le trouvez toujours, dans ses conversations avec les sauvages, occupé à catéchiser. On peut lui attribuer une part dans la conversion du chef souriquois Membertou, en Acadie, "le plus méchant et traître, dit-il, qui fut entre ceux de sa nation ». Membertou dépassait les cent ans à son baptême, et son fils aîné, baptisé avec lui, en avait soixante. Quant à son plus intime ami, le chef algonquin Yroquet, c'est bien Champlain en personne qui l'amena à la foi, autant par l'illumination de la bonté que par celle de l'Esprit-Saint; la conversion alla si loin qu'Yroquet osait intervenir auprès de ses guerriers pour empêcher le supplice des prisonniers ennemis, car la réciprocité de barbarie était entière entre Iroquois et sauvages alliés des Français. L'hiver qu'il passa en Huronie, Champlain s'attacha le grand chef Darontal; on ne dit pas qu'il l'ait converti, mais il lui expliquait à loisir, peut-être un peu maladroitement, le mystère chrétien. Le Huron répondait : a Tu dis des choses qui surpassent notre esprit et nous ne pouvons comprendre par discours. Mais si tu désires que nous les sachions, il est nécessaire d'amener en ce pays femmes et enfants, afin que nous apprenions la façon de vivre que tu mènes, comme tu adores ton Dieu, comme tu obéis aux ordres de ton roi... Car voyant ces choses, nous apprendrons plus en un an qu'en vingt, jugeant notre vie misérable au prix de la tienne. » La théologie de l'exemple! Ces bons sauvages en connaissent la vertu. Et Dieu sait si Champlain leur versait aussi cette doctrine! A Québec même, c'est le chef montagnais Miristou qui suit Champlain comme son ombre; il lui demande même la permission de l'appeler son frère pour bien lui marquer son attachement.

Mais ce ne sont là que des initiatives personnelles. Il fallait surtout créer des institutions.

La fondation de l'Eglise canadienne coïncide avec l'arrivée des premiers missionnaires, en 1615. Il y avait donc fallu sept ans. Champlain était allé chercher ces ouvriers aux Etats généraux de France, l'année précédente, où il avait obtenu l'agrément du prince de Condé, alors vice-roi de la Nouvelle-France, et de tous les cardinaux et évêques assemblés, ainsi que "des aumônes de près de 1,500 livres " (environ trois cents dollars). C'est le 25 juin 1615, en la chapelle des Franciscains Récollets, placés sous le vocable de l'Immaculée-Conception, que fut célébrée la première messe, en présence du gouverneur. Les Récollets étaient au nombre de quatre, trois Pères et un Frère. La paroisse de Québec existait, cellule-mère de toutes celles qui allaient plus tard 
constituer le bastion de notre foi et aussi de notre survivance ethnique. On essaya d'adjoindre au couvent une petite école pour les enfants sauvages; cinq ou six $\mathrm{y}$ entrèrent et en sortirent aussi vite... Mais les missions s'organisèrent : à Tadoussac, aux Trois-Rivières, chez les Hurons et chez la Nation Neutre. Toutefois, le fruit n'était pas consolant.

Les Récollets se sentaient trop pauvres, de ressources matérielles et d'influences. Ils appelèrent eux-mêmes à la rescousse un Ordre que favorisait son prestige à la cour depuis Henri IV : la Compagnie de Jésus.

Champlain se rendit négocier en France cette importante affaire. Le duc de Ventadour, qui venait d'être nommé vice-roi, avait justement pour directeur spirituel un Jésuite, lui-même enflammé depuis longtemps pour les missions du Canada. Les négociations ne devaient pas traîner. Le duc les prit en mains, fit approuver le projet par le roi et Richelieu, s'offrit à faire lui-même les frais des six premiers fils d'Ignace et enjoignit aux directeurs de la compagnie de commerce de prendre les frais d'établissement bon gré mal gré. Et sur ce bateau de 1625, Champlain avait à côté de lui l'Hercule des missions du NouveauMonde, qui allait donner vingt-quatre ans de sa vie et sa vie même à cette œuvre : Jean de Brébeuf. Les Jésuites partagèrent d'abord les œuvres avec les Récollets. Puis, en 1632, après les trois années d'occupation anglaise, ils eurent seuls la charge de toute l'Eglise de la colonie. C'était une forte armature. Après Dieu, il faut en donner le mérite au Père de la Nouvelle-France.

En fin de compte, si les arbres n'avaient pas encore donné beaucoup de fruit quand s'éteignit le planteur Champlain, du moins ils avaient été plantés et de grandes racines commençaient à s'étendre dans le sol en même temps que quelques rameaux au-dessus de terre.

Comme je veux finir sur la note héroïque, et d'autre part, faire un léger éclat en l'honneur de ma mère l'Eglise, l'Eglise de mon pays, je vais citer un historien récent des colonies françaises, de la colonie canadienne en particulier. Il s'agit de Claude de Bonnault, auteur d'une Histoire du Canada français, publiée en 1950 (P.U.F.), donc pas tellement loin de l'ère scientifique, ce qui sera apprécié, je l'espère, par les historiens dits scientifiques. Voici comment parle cet homme qui n'a pas de raisons de famille d'être apologétique *:

«Franchement y a-t-il un seul moment des origines canadiennes qui ne témoigne du plus authentique héroïsme?

Le Canada est né dans une atmosphère de légende et d'épopée; dans un climat inhumain, il a souffert; dans une ambiance surnaturelle il a grandi. En aucun temps, dans un pays, s'est-il vu triomphe de l'esprit, victoire de l'âme sur le corps, comparables à ce que fut la colonisation du Canada ou Nouvelle-France ? A-t-on connu ailleurs plus sublime exaltation de foi et de charité ?

La colonisation du Canada n'a pas été une colonisation comme les autres. Elle a été l'œuvre d'une élite. Parce que seule une 
élite pouvait jeter un tel défi aux forces physiques. Ce fut une partie désespérée, engagée contre toutes les résistances de la nature et des hommes. Aux yeux du monde, absurdité que cette entreprise, pareille à la folie de la croix ou plutôt folie même de la croix. $\gg$ (p. 9)

Le même universitaire français dira encore : "Les missionnaires du Canada étaient des saints. Quelques-uns ont été canonisés. Presque tous devraient l'être. Oui, des saints que ces hommes. Ft des héres, les laïques qui les avaient prórédé́a. (n. 13)

Serait-il exagéré d'affirmer que, parmi toutes ces grandes âmes, celle de Champlain fut une des plus magnifiques? Incontestablement, Champlain est un modèle de saint bâtisseur, aimant Dieu et sa patrie, que la jeunesse canadienne-française devrait mieux connaître. Il est un exemple parmi tant d'autres de la virilité et de la grandeur de nos ancêtres. Me sera-t-il permis de souhaiter qu'un autre Champlain nous soit donné pour fonder, cette fois, une nouvelle Nouvelle-France, c'està-dire un Québec rénové, puissant, libéré de ses chânes, véritablement français et authentiquement catholique?

Raymond BARBEAU 